\title{
IdeAs
}

Idées d'Amériques

$17 \mid 2021$

Villes et culture dans les Amériques

\section{Questions à Stéphan La Roche, PDG du Musée de la Civilisation (Québec)}

Diana Burgos-Vigna et Cynthia Ghorra-Gobin

\section{OpenEdition}

1 Journals

Édition électronique

URL : https://journals.openedition.org/ideas/10120

DOI : $10.4000 /$ ideas. 10120

ISSN : 1950-5701

Éditeur

Institut des Amériques

Référence électronique

Diana Burgos-Vigna et Cynthia Ghorra-Gobin, « Questions à Stéphan La Roche, PDG du Musée de la Civilisation (Québec) », IdeAs [En ligne], 17 | 2021, mis en ligne le 01 mars 2021, consulté le 05 juin 2021. URL : http://journals.openedition.org/ideas/10120; DOI : https://doi.org/10.4000/ideas.10120

Ce document a été généré automatiquement le 5 juin 2021

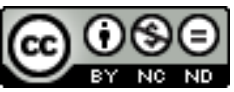

IdeAs - Idées d'Amériques est mis à disposition selon les termes de la licence Creative Commons Attribution - Pas d'Utilisation Commerciale - Pas de Modification 4.0 International. 


\title{
Questions à Stéphan La Roche, PDG du Musée de la Civilisation (Québec)
}

\author{
Diana Burgos-Vigna et Cynthia Ghorra-Gobin
}

\section{Musée et territoire}

\begin{abstract}
DiANA BURgos-vignA ET CYNTHIA GHORRA-GOBIN : Notre dossier s'attache à la place de la Culture dans les villes. Aussi nous souhaiterions avoir votre opinion sur le rôle que remplit le Musée de la Civilisation dans la province de Québec, dans un premier temps, et dans la ville de Québec dans un second temps. Quelle différence faites-vous en termes de rayonnement entre le Musée et le complexe muséal (incluant 4 sites dont 2 musées)?
\end{abstract}

Stéphan La Roche : Le Musée de la civilisation est un musée national, donc celui de tous les Québécois, avec qui il entretient d'ailleurs une histoire d'amour depuis son ouverture en 1988. Nous sommes un musée de société qui évolue avec celle-ci et qui doit donc rester agile pour suivre cette évolution (et parfois même pour tenter de devancer la société en la "brassant » un peu). En fait, nous sommes un musée qui a l'audace dans son ADN, et ce, depuis plus de trente ans.

Nous avons été précurseur d'une « nouvelle » muséologie, beaucoup plus interactive et immersive, qui cherche à s'adresser à différents niveaux de public et se donnant un rôle social en cherchant à susciter la réflexion. Ce rôle social, cette action citoyenne, se traduisent notamment par des expositions ou des activités visant l'intégration des nouveaux arrivants. La culture autochtone est aussi très présente dans la programmation, en lien avec l'exposition permanente C'est notre histoire.

Nous sommes également un pôle d'expertise en matière de muséologie et de conservation préventive avec notre Centre national de conservation et d'étude des collections. En étant le gardien de la mémoire collective, une mission nationale de préservation du patrimoine qui ne concerne pas uniquement les objets qui marquent l'histoire, mais aussi la mémoire des courants sociaux et culturels.

Nous sommes enfin et surtout un musée fortement identitaire. Notre programmation est jalonnée d'expositions déclinant de diverses facettes de l'identité québécoises 
(Mémoires (1988), Je vous entends chanter (1996), Téléromans (1997), Fou de hockey (1998), Cinéma (2006), Sortir de sa réserve (2018), Broue - L'homme des tavernes (2019), Histoires de pêche (2020).

\section{...et dans la ville de Québec dans un second temps :}

Le Musée est une des institutions phares de la ville de Québec, bien connue des citoyens, qui en sont fiers, qui l'ont souvent fréquenté lorsqu'ils étaient enfants, et qui y reviennent avec leurs propres enfants. Le Musée de la civilisation est une agora, un lieu d'échanges et de partage de contenus pour la communauté, qui s'y retrouve pour toutes sortes d'activités culturelles, éducatives, sociales, économiques ou touristiques.

Notre musée est aussi un phare dans la ville en terme architectural avec notre campanile lumineux qui est un repère urbain. Nous sommes évidemment gardien du patrimoine puisque l'architecture audacieuse de notre institution intègre la Maison Estèbe (xviII ${ }^{\text {e }}$ siècle) permettant au passé et au présent de cohabiter harmonieusement.

Avec le temps, nous avons développé un volet de programmation qui offre une vitrine pour des sujets plus locaux. Par exemple, nous soulignons les anniversaires d'institutions locales (par exemple : Grand Théâtre) ou de moments marquants de l'histoire de la Ville (création des Services de police et de protection contre les incendies, fondation de la Chambre de commerce et d'industrie de Québec) grâce à des expositions et activités qui mettent en avant ce pan de l'évolution de notre ville, en concertation avec les organisations concernées.

D.B.V. ET C.G.G. : Quelle différence faites-vous en termes de rayonnement entre le Musée et le complexe muséal (incluant 4 sites dont 2 musées)?

S.L.R. : Depuis son ouverture en 1988, notre musée s'est vu confier divers mandats additionnels et complémentaires par l'État québécois. Le principal ajout consiste en la gestion du site patrimonial et des collections encyclopédiques du Séminaire de Québec, première institution d'enseignement en Nouvelle-France et première université francophone en Amérique du Nord. Un musée portant sur l'Amérique française existait déjà sur le site au moment de notre prise en charge en 1995. Ce musée est maintenant à un tournant puisque nous sommes à mener un grand projet de transformation de ce site pour l'articuler davantage autour de l'histoire de ce site fondateur de la Nouvelle-France (et donc du Québec d'aujourd'hui) et, de là, parler des fondements de notre identité. Il s'agit donc d'un rôle complémentaire à celui du Musée de la civilisation.

Nos deux autres sites sont voués à la conservation. Le Centre national de conservation et d'études des collections ( $\mathrm{CNCEC}$ ) est notre grande réserve muséale où conditions optimales de conservation servent de référence dans le secteur muséal international. Enfin, le site patrimonial de Place-Royale et la Maison historique JeanBaptiste Chevalier constituent des points d'ancrage d'un parcours historique de notre ville.

\section{Musée et ville}


D.B.v. ET C.G.G. : Le musée a-t-il une politique spécifique à destination des habitants de la ville de Québec ? Et si oui, quelles sont les actions menées pour toucher ce public ?

S.L.R. : C'est la signature particulière de notre institution que d'être capable de s'allier à un public qui traverse les générations, tant le contenu se déploie sur différentes " couches " de sens et de facettes. Chaque résident de Québec a son souvenir du Musée. Notre «politique» passe par notre action culturelle, notre engagement citoyen, des partenariats avec des groupes et des institutions culturelles locales. Cela passe aussi par une journée d'accès gratuit pour tous, le premier dimanche de chaque mois.

D.B.V. ET C.G.G. : Quel sens donnez-vous à l'expression « musée populaire » ? Cela renvoie-til au principe d'une volonté de transmission de connaissances produites par des chercheurs spécialisés vers le grand public ?

S.L.R. : Nous sommes fiers de nous définir comme un musée populaire, au sens noble du mot. C'est à dire un musée accessible à tous, peu importe l'âge, la condition sociale, l'origine. Nous plaçons l'être humain au cœur de notre mission et au centre de nos préoccupations. Nous voulons bien sûr lui transmettre des connaissances, mais nous voulons aussi le faire réfléchir et lui faire faire vivre des émotions, lui faire toucher l'enchantement et l'émerveillement.

Par exemple, nous nous attachons à présenter nos collections selon des points de vue multiples pouvant rejoindre le plus grand nombre; nous utilisons des moyens muséographiques diversifiés incluant les interactifs donnant accès aux contenus sous différentes formes ; notre muséographie est très scénarisée pour susciter l'intérêt et faire vivre des émotions.

D.B.v. ET C.G.G. : Le Musée a-t-il des relations particulières avec d'autres institutions de la ville, et si oui lesquelles?

S.L.R. : Notre musée mène des projets avec de multiples organisations de notre ville. Mentionnons la Commission de la Capitale-Nationale du Québec pour la mise en valeur du site archéologique Cartier-Roberval et l'animation de la place des Canotiers. Nous avons aussi programmé avec eux pendant de nombreuses années des séries de conférences sur l'histoire de notre ville.

Nous collaborons de plus en plus avec notre institution sœur dans la ville : le Musée national des beaux-arts du Québec (MNBAQ) avec lequel nous avons développé des programmes d'abonnements conjoints et des activités de promotion croisées. Nous amorçons avec le MNBAQ la mise en place d'un projet structurant autour de l'innovation et de la créativité afin de permettre à nos équipes respectives d'échanger sur leurs expertises en matière de mise en valeur des collections, de médiation et d'expérience du visiteur et de projets numériques.

Enfin, notre programmation d'activités culturelles au Musée de la civilisation (2 auditoriums et le grand hall) ainsi que la Chapelle historique du Séminaire de Québec nous offre de nombreuses occasions de bâtir des projets artistiques (concerts, spectacles, lectures, etc...) avec l'ensemble des organisations culturelles de la ville, de l'orchestre symphonique aux compagnies de théâtre de marionnettes, en passant par l'Université Laval et ses différentes facultés. 


\section{Relations avec la France}

D.B.v. ET C.G.G. : Vous avez un parcours international et avez passé plusieurs années en France. En quoi ce passage par la France a-t-il influencé votre vision de la culture et / ou des politiques culturelles que vous menez au Québec?

S.L.R. : J'ai occupé les fonctions de directeur des Services culturels à la Délégation générale du Québec à Paris de janvier 2001 à juillet 2004. Mon rôle était de planifier et diriger les services culturels offerts par la Délégation générale du Québec à Paris sur le territoire français pour l'ensemble des disciplines artistiques et des secteurs culturels dans une optique de rayonnement. En collaboration avec de multiples partenaires québécois et français, nous devions élaborer des stratégies d'action visant la promotion de la culture québécoise en France. J'ai pu notamment contribuer à la mise en place de la première Entente France-Québec en matière de patrimoine, muséologie et archives.

Il est évident que cette expérience, en plus de me permettre de développer des liens avec de nombreux partenaires sur le territoire français et constater à quel point notre culture sait voyager, m'aura permis d'aiguiser cette capacité à concevoir la culture québécoise au-delà des frontières, la positionner dans la grande Histoire du Monde, des sociétés, ceci totalement en phase avec la mission de notre Musée.

Né à Québec, je suis très attaché à ma ville et aux institutions qui m'ont formé. C'est en vivant à Paris que j'ai pris la pleine mesure de cet attachement. S'ouvrir sur le monde, c'est aussi redéfinir la notion d'ancrage et d'identité. Et c'est précisément ce que nous cherchons à faire au Musée de la civilisation.

D.B.v. ET C.G.G. : $Y$ a-t-il des liens entre le Musée de la Civilisation et des institutions culturelles françaises ? Pouvez-vous notamment détailler la nature du partenariat avec la Cité des Sciences à la Villette?

S.L.R. : Les liens entre le Musée de la civilisation et les institutions françaises sont nombreux et présents depuis nos débuts. Nos collaborations concernent tant le Musée des Confluences de Lyon (Corps rebelles et Venenum), le MUCEM de Marseille (Pharaons superstars) le Muséum national d'histoire naturelle (et le Musée de l'homme), le musée du Quai Branly; nous avons aussi collaboré avec d'autres institutions comme le Musée Dauphinois de Grenoble. Soulignons aussi notre adhésion au réseau des Micro-Folies, initié par le Parc de La Villette, en 2018.

Sur le même site de La Villette, la Cité des Sciences et de l'Industrie de Paris et donc Universcience, est un partenaire avec lequel nous comptons déjà de nombreuses collaborations, dont Chiens et chats en 2016 et l'exposition Effets spéciaux, cette année. Clairement il y a une belle parenté entre nos deux institutions, avec des approches et des contenus qui se rejoignent beaucoup. Nous comptons également échanger sur nos projets respectifs de rénovation et transformation du Palais de la découverte à Paris et la Cité du Séminaire, à Québec qui sont tous deux prévus pour 2024.

Enfin, nous amorçons en ce moment une collaboration avec le Musée du Louvre afin d'échanger sur nos expertises en matière de collections, de politiques culturelles et d'innovations muséographiques, grâce au soutien de l'Accord France-Canada sur les musées. 


\section{Le musée et ses publics}

D.B.v. ET c.G.G. : Comment concevez-vous le rôle des musées dans la société actuelle caractérisée par de fortes inégalités d'accès à la culture et par l'emprise grandissante du numérique dans tous les secteurs y compris la culture?

S.L.R. : Cette question est au cœur de notre action. Par exemple, nous avons lancé, il y a environ un an, un projet nommé Sésame, qui, comme son nom l'indique, vise une plus grande ouverture sur diverses communautés, notamment les personnes handicapées, les personnes en situation d'itinérance ou les personnes en provenance de l'immigration. Nous menons des démarches individualisées avec ces groupes dans un double objectif, celui de mieux les connaître afin de mieux les représenter dans nos expositions et activités et afin qu'ils nous apprivoisent et se sentent les bienvenus dans nos espaces.

Nous sommes très engagés dans la communauté afin d'en assurer l'accessibilité à toutes les personnes, peu importe leur milieu, par le biais de la Fondation du Musée et son action philanthropique : accueil de 6000 personnes de milieux défavorisés et une moyenne de 100000 enfants de milieux plus précaires se voient ouvrir les portes du Musée gracieusement dans un cadre valorisant.

Notre Fondation a mis en place depuis deux ans une nouvelle approche philanthropique misant sur les jeunes adultes de 18 à 35 ans pour en faire des ambassadeurs du Musée et appelée Génération M. Nous sommes proactifs pour faire de la philanthropie l'affaire de toutes les générations dans le but de perpétuer cet attachement à la culture et sa reconnaissance en tant que levier de développement social chez les générations qui suivent. Génération $M$ parraine maintenant des journées spéciales où nous accueillons au Musée de la civilisation tous les élèves d'une même école en même temps avec une programmation qui a été conçue pour eux. Notre action va jusque-là !

Pour ce qui est de la place grandissante du numérique : sujet d'actualité s'il en est un, plus présent que jamais dans nos vies, le numérique demeure par ailleurs un vecteur de développement utile aux communautés, aux entreprises et aux individus. L'intérêt pour ces questions se confirme notamment par la popularité de notre laboratoire d'expérimentations numériques, le MLab Créaform, qui s'est démarqué par de nombreux prix et mentions au cours de cette année et qui remporte un impressionnant succès auprès des jeunes et des moins jeunes depuis son ouverture. Les musées doivent se servir du numérique pour pousser leur mission encore plus loin, innover tout en demeurant pertinents. Nous avons jugé pertinent d'en faire le sujet d'une grande exposition intitulée La Tête dans LE nuage qui explore la révolution numérique et met en relief son impact sur nos comportements individuel, interpersonnel et collectif, questionnant le présent pour nous projeter dans l'avenir, suscitant par le fait même certaines prises de conscience.

D.B.v. ET c.G.G. : Comment voyez-vous leur évolution dans un contexte marqué par des changements dans les pratiques culturelles?

S.L.R. : Le Musée de la civilisation, depuis ses tout débuts, œuvre à la démocratisation de la culture et à favoriser le contact de publics de tous horizons avec celle-ci par des contenus riches, éducatifs, de grande qualité : cette mission se poursuit par les gestes novateurs que le numérique permet. Les projets cités plus haut en sont de beaux 
exemples, annonciateurs des opportunités qui s'offriront dans le futur. Tous les musées seront appelés à se transformer. Le numérique est là pour rester et transformera pour de bon nos façons de faire.

D.B.V. ET C.G.G. : Et quelles mesures peuvent être mises en place pour toucher les publics de tous âges et de toutes origines?

S.L.R.: C'est un défi sans cesse renouvelé, mais fort stimulant. Par exemple, pour rejoindre les personnes âgées nous avons mis en place il y a quelques années le programme Réminiscence. Quand le passé aide le présent qui permet de rejoindre les aînés en perte d'autonomie dans leurs résidences en leur faisant revivre l'émotion de la visite d'un lieu qui a marqué la jeunesse de presque tous : le magasin général. Sous un autre angle, nos collections en ligne permettent, depuis 2017, à tous les publics de s'amuser à concevoir des albums ou des mosaïques d'images d'objets de collection, tout en apprenant et découvrant des pans de notre histoire. Ces questions font partie de nos orientations stratégiques au cœur même de notre gouvernance et du développement de notre offre.

D.B.v. ET C.G.G. : Que faut-il entendre aujourd'hui par «médiation culturelle»?

S.L.R. : En synthétisant beaucoup, la médiation culturelle c'est la vulgarisation de contenus pour rejoindre des clientèles spécifiques. Aujourd'hui, il faut saisir les opportunités de mise en valeur de contenus nouveaux permettant une présence étendue dans la durée et sur le territoire. En ce sens, les outils numériques sont en train de transformer la médiation culturelle en nous permettant de diffuser nos contenus partout sur notre territoire et même dans le monde entier et ce, pour une longue période. La médiation, qu'elle se fasse in situ ou de manière virtuelle, c'est engager une conversation avec des publics variés, en utilisant notre offre comme prétexte. L'objectif, c'est l'échange, le partage. Et c'est ce qui est si beau avec la culture : quand elle se partage, elle se multiplie. Celui qui la donne ne la perd pas, celui qui la reçoit en est plus riche. Pour nous au Musée de la civilisation, c'est ça la médiation culturelle.

\section{Musée et pandémie}

D.B.v. ET C.G.G. : Quel a été l'impact de la pandémie sur la politique du musée? Avez-vous anticipé sur la réaction du public ? et quels effets à long terme sur la stratégie mise en place en lien avec les publics?

S.L.R.: La pandémie a eu, comme chacun sait, de multiples effets sur nos organisations. D'abord à l'interne : l'implantation du télétravail à grande échelle, le déploiement d'équipements technologiques chez les employés, le défi de la communication avec les équipes que nous avons relevé grâce à des courriels quotidiens et des capsules vidéo hebdomadaires du PDG transmises à l'ensemble des employés, l'implantation d'un nouvel Intranet.

Par rapport aux publics, nous avons réagi très rapidement en mettant en place deux initiatives complémentaires. Nous avons créé un nouveau site web sous l'appellation Une heure au musée avec dix partenaires culturels de partout au Québec et nous avons lancé l'opération Documentez la pandémie pour recueillir des témoignages, photos et dessins ainsi que des objets provenant des citoyens afin de documenter pour les générations futures cette période historique de confinement que nous vivons. 
$\mathrm{Au}$ moment où les citoyens avaient encore plus besoin de partager un «vivre ensemble », notamment par la culture et les arts, le Musée de la civilisation a ainsi rapidement voulu participer le plus activement possible au mieux-être de la collectivité. En cette période de confinement, la visite virtuelle des institutions muséales demeure l'alternative par excellence pour se divertir, s'instruire, se documenter, grandir personnellement et collectivement. Nous avons donc offert avec Une heure au Musée, une plateforme conviviale de recension de contenus muséaux (visites virtuelles, activités pour les groupes scolaires, conférences, jeux, etc.), accessibles en ligne et destinés à toute la famille, à partir de laquelle nous avons également invité le public à nous aider à documenter la pandémie, mission que nous nous sommes donnés. En cette période de pandémie, les technologies numériques nous auront permis d'offrir la culture au plus grand nombre, facilement, rapidement et gratuitement.

\section{AUTEURS}

\section{DIANA BURGOS-VIGNA}

Diana Burgos-Vigna est professeure en études latino-américaines à l'Université Paris Nanterre et membre du laboratoire CRIIA où elle est responsable du GRECUN (Groupe État Culture Nation dans le monde ibéro-américain). Ses recherches portent principalement sur les villes sudaméricaines et s'articulent autour de trois axes principaux : la démocratie participative, les réseaux de villes, et les politiques culturelles et patrimoniales locales.

\section{CYNTHIA GHORRA-GOBIN}

Cynthia Ghorra-Gobin CNRS-Iheal, Université Sorbonne Nouvelle, est géographe et membre du laboratoire CREDA (Centre de recherche et de documentation des Amériques). Ses recherches qui portent principalement sur les villes nord-américaines sont centrées sur les transformations spatiales et culturelles de la ville en relation avec la mondialisation et la globalisation. Elle a coordonné les deux éditions du Dictionnaire critique de la mondialisation, Armand Colin, 2006 et 2012. 